Check for updates

Cite this: RSC Adv., 2019, 9, 19675

Received 20th March 2019 Accepted 18th June 2019

DOI: 10.1039/c9ra02134e

rsc.li/rsc-advances

\section{Facile synthesis of exfoliated vermiculite nanosheets as a thermal stabilizer in polyvinyl chloride resin}

\author{
Weiliang Tian, ${ }^{\star a b}$ Zhong Li, ${ }^{a}$ Kewei Zhang (D) *acd and Zhenhong Ge ${ }^{a}$
}

Well-defined vermiculite (VMT) nanosheets have been exfoliated from mineral VMT by a water-assisted anion-exchange approach. The resultant VMT nanosheets are utilized as a thermal stabilizer in polyvinyl chloride (PVC) to resist dehydrochlorination of PVC. As expected, the color-blackening onset of PVC is delayed after incorporating VMT nanosheets, and the thermal stability improves with reducing the particle size of the VMT nanosheets. The dehydrochlorination temperature appears to be increased by $13{ }^{\circ} \mathrm{C}$ for the PVC resin with $6 \mathrm{wt} \%$ VMT additive. The improved thermal stability is attributed to the negatively charged laminates of VMT that stabilize hydrogen chloride produced from thermal degradation of PVC. This work not only provides a cost-effective approach to prepare VMT nanosheets but also presents new insight towards the design of thermal stabilizers.

\section{Introduction}

Polyvinyl chloride (PVC), a type of chlorine-containing plastic, is widely used in our daily life due to its general versatility, low cost, high stability, and non-flammable feature. ${ }^{1,2}$ However, it suffers from thermal dehydrochlorination with extended use, ${ }^{3,4}$ which leads to color darkening, performance degradation and even health hazards. Therefore, the stabilization of PVC products by incorporating a thermal stabilizer has been the subject of much research. So far, a trend in thermal stabilizers including metal alkoxides, ${ }^{5}$ organo complexes, ${ }^{3}$ rare-earth compounds, ${ }^{6}$ and hydrotalcite-like materials ${ }^{7}$ has been developed. However, these thermal stabilizers are generally expensive, limiting their application in the manufacture of industrial PVC products. It remains a great challenge to search for an inexpensive and environmentally friendly thermal stabilizer for PVC.

Vermiculite (VMT) is a highly-charged natural clay, showing a triclinic structure of tetrahedral-octahedral-tetrahedral aluminosilicate layers and octahedrally coordinated $\mathrm{Mg}^{2+}, \mathrm{Ca}^{2+}$ and $\mathrm{K}^{+}$in the interlayer space. ${ }^{8}$ Owing to its chemical and mechanical stability, the layered VMT has been widely used in

\footnotetext{
${ }^{a}$ Key Laboratory of Chemical Engineering in South Xinjiang, College of Life Science, Tarim University, Alar 843300, P. R. China.E-mail: twllong@126.com

${ }^{b}$ State Key Laboratory of Organic-Inorganic Composites, Beijing University of Chemical Technology, Beijing 100029, P. R. China

${ }^{c}$ Guangxi Key Laboratory of Petrochemical Resource Processing and Process Intensification Technology, Guangxi University, Nanning 530004, P. R. China

${ }^{d}$ State Key Laboratory of Bio-fibers and Eco-textiles, Collaborative Innovation Center of Shandong Marine Biobased Fibers and Ecological Textiles, College of Materials Science and Engineering, Institute of Marine Biobased Materials, Qingdao University, Qingdao 266071, P. R. China.E-mail: zhkw@qdu.edu.cn
}

a variety of applications, including soil conditioner, ${ }^{9}$ gas barrier, ${ }^{10,11}$ adsorption of cations, ${ }^{12}$ and high-temperature refractory insulation of polymers. ${ }^{13,14}$ Well-defined twodimensional (2D) characteristic is beneficial for VMT to exhibit superior properties. Thus, great effort has been devoted to exfoliating VMT nanosheets. Nowadays, the exfoliating strategies of VMT nanosheets mainly focus on thermal intumescing, which is unsatisfied concerning the thickness of nanosheets. It is desirable to exfoliate few-layer VMT nanosheets under mild and low-cost conditions.

In the present work, a facile water-assisted anion-exchange approach has been demonstrated to realize the cost-effective exfoliation of few-layer VMT nanosheets from nature mineral. The layered VMT is utilized as an effective PVC thermal stabilizer. By adding VMT nanosheets in PVC resin, a perfect thermal stability of the PVC is accomplished. The improvement of thermal stability is discussed through thermogravimetric analysis. Substantially, the VMT nanosheets possess negatively charged laminates and metal cations between adjacent layers, which could stabilize hydrogen chloride $(\mathrm{HCl})$ produced from the thermal degradation of PVC. This work provides a novel strategy to realize thermal stabilization of polyvinyl chloride by adding VMT nanosheets.

\section{Experimental}

\section{Preparation of VMT dispersion}

In a typical procedure, $1.0 \mathrm{~g}$ of $\mathrm{NaCl}$ and $1.0 \mathrm{~g}$ of VMT were dissolved into $5 \mathrm{~mL}$ of deionized water, which was vigorously smashed at a speed of $400 \mathrm{rpm}$ for $4-72 \mathrm{~h}$ at room temperature. Then, the product was washed with deionized water to remove ions possibly remnant in the products and collected by 
centrifugal separation under different speeds, including $4000 \mathrm{rpm}, 6000 \mathrm{rpm}, 8000 \mathrm{rpm}$ and $10000 \mathrm{rpm}$.

\section{Preparation of PVC-VMT films}

In a typical procedure, the VMT nanosheets were dispersed in ethanol under ultrasound, and then the PVC powder was added in the dispersion. After fully mixed by sonication, the VMT-PVC paste was dried at $105^{\circ} \mathrm{C}$ to form uniform mixture. The mixture was mixed with 50 parts of dioctylphthalate (DOP) per hundreds of resin in a beaker, stirred at $3500 \mathrm{rpm}$ for $30 \mathrm{~min}$ at $60{ }^{\circ} \mathrm{C}$, and then gelated at $110{ }^{\circ} \mathrm{C}$ for $10 \mathrm{~min}$. Finally, the resulting composite was molded to film with a thickness of $1.0 \mathrm{~mm}$ and cut into strips of $3 \mathrm{~cm} \times 2 \mathrm{~cm}$.

\section{Characterization}

The product morphology was examined by field emission scanning electron microscopy (SEM, Zeiss Supra 55) and highresolution transmission electron microscopy (HRTEM, JEM2100). Crystallographic information was collected using powder X-ray diffraction (XRD, Shimadzu 6000) in the range of $5-70^{\circ}(2 \theta)$. The Fourier transform infrared (FTIR) spectra were obtained using a Vector 22 (Bruker) spectrophotometer in the range of $400-4000 \mathrm{~cm}^{-1}$ at room temperature.
Thermogravimetric analysis was performed in air with a heating rate of $10{ }^{\circ} \mathrm{C} \min ^{-1}$ using a NETZSCH instrument (STA 449 F5 Jupiter®).

\section{Measurements}

The discoloration tests of the PVC films were operated according to the standard of ISO 305:1990(E). The specimens were placed in a thermal aging test oven at $180{ }^{\circ} \mathrm{C}$. After static thermal aging, the specimens were taken out, and the color of the specimens was recorded every $10 \mathrm{~min}$. The Congo red tests were conducted to evaluate the thermal stability time of PVC, according to ISO 182/1-1990. A certain amount of PVC was put into a tube with the Congo red test paper located at $3 \mathrm{~cm}$ above the sample. The test tube was kept at $180{ }^{\circ} \mathrm{C}$ in an oil bath. The thermal stability time was determined by the time of the color change (from red to blue) on Congo red paper.

\section{Results and discussion}

Fig. 1a depicts the exfoliating process of VMT nanosheets by a water-assisted anion-exchange approach. The mineral VMT (Fig. 1b) is firstly dispersed in $\mathrm{NaCl}$ aqueous slurry. After sonication and ball milling, the sodium cations and water
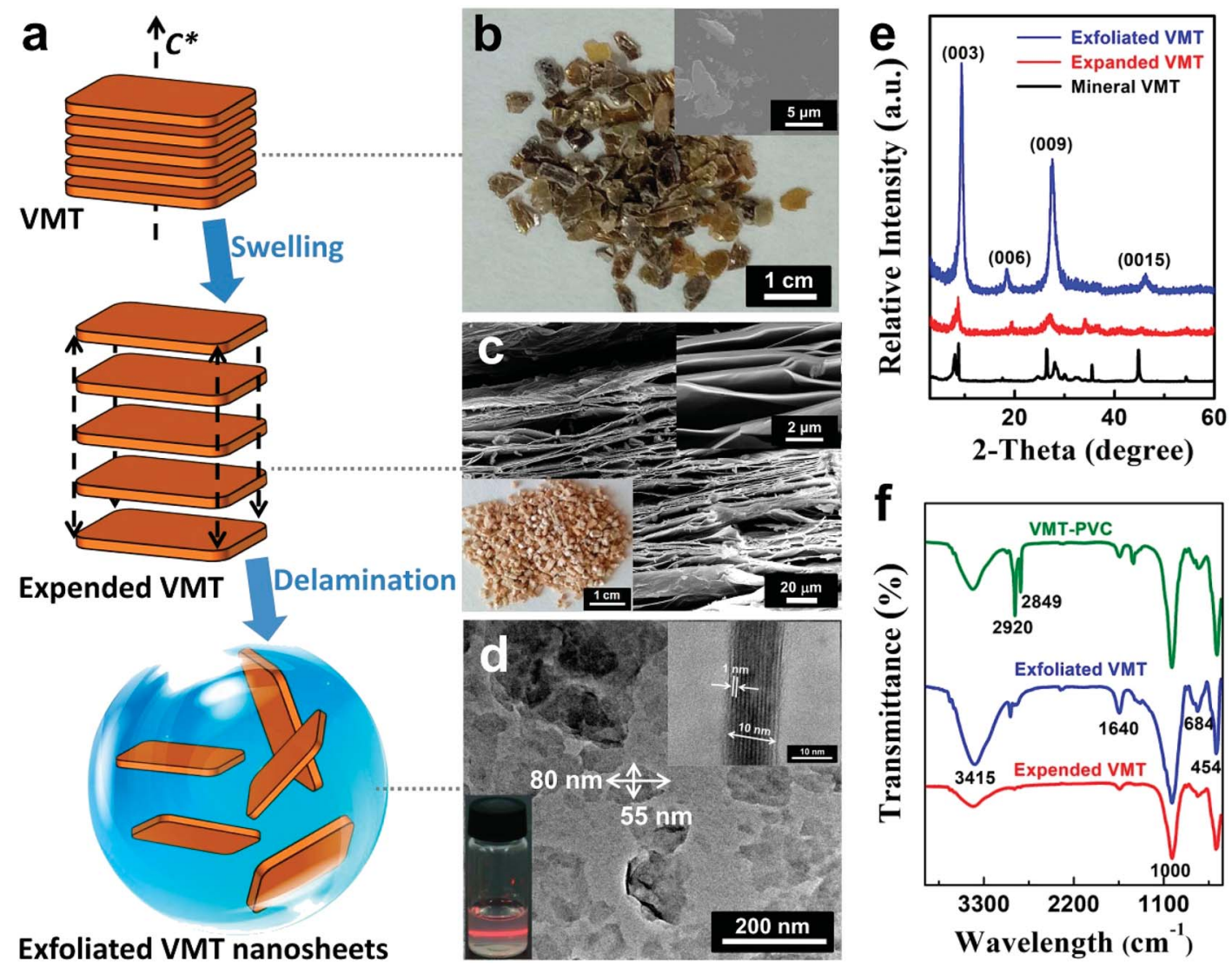

Fig. 1 (a) Schematic illustration of the exfoliating process. (b) Optical photograph of mineral VMT. Inset shows SEM image of mineral VMT. (c) SEM image of expanded VMT. Insets show enlarged SEM view and optical photograph of expanded VMT. (d) TEM image of exfoliated VMT. Insets show enlarged view of a representative nanosheet and the Tyndall phenomenon of the nanosheet dispersion. (e) XRD patterns of mineral VMT, expanded VMT and exfoliated VMT. (f) FTIR spectra of the expanded VMT, exfoliated VMT and VMT-PVC composite. 


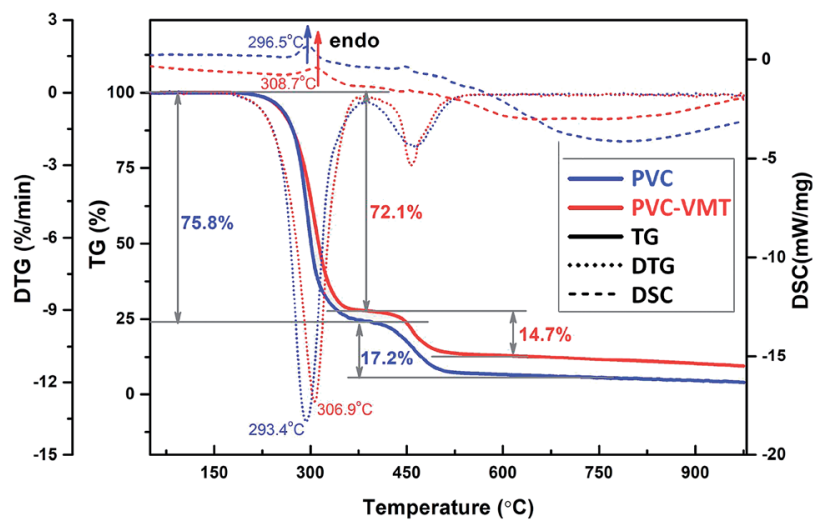

Fig. 2 Thermal curves of TG, DTG and DSC for the pure PVC and VMT-PVC composite.

molecules infiltrate into VMT layers, resulting in expanded VMT. The expanded VMT is in millimeter, possessing multilayered structures, as characterized by the SEM image (Fig. 1c). After delamination in aqueous solution, the densely packed multilayers are exfoliated to thin layers with size of 50$200 \mathrm{~nm}$, thickness of $\sim 10 \mathrm{~nm}$ and inter-planar distance of $\sim 1 \mathrm{~nm}$, as characterized by the TEM image (Fig. 1d). Besides, the stable colloidal solution of the exfoliated VMT layers shows a clear Tyndall light scattering (lower left inset of Fig. 1d). As shown in Fig. 1e, the (003) reflection peak of VMT shifts from $2 \theta$ $=8.64^{\circ}(1.02 \mathrm{~nm})$ to $2 \theta=9.31^{\circ}(0.95 \mathrm{~nm})$ after swelling and delamination, confirming the reduced inter-planar distance of VMT nanosheets. ${ }^{15}$ Besides, the XRD profiles become stronger and sharper, indicating improved crystallinity and purity of the exfoliated VMT. In FTIR spectra (Fig. 1f), the peaks at $454 \mathrm{~cm}^{-1}$,
$684 \mathrm{~cm}^{-1}$ and $1000 \mathrm{~cm}^{-1}$ are ascribed to $\mathrm{Si}-\mathrm{O}, \mathrm{Al}-\mathrm{O}$ and $\mathrm{Si}-\mathrm{O}$ vibration, ${ }^{16}$ respectively, while the peaks at $1640 \mathrm{~cm}^{-1}$ and $3415 \mathrm{~cm}^{-1}$ are assigned to $-\mathrm{OH}$ bending and stretching vibration of weakly adsorbed water on the surface. ${ }^{17}$ The FTIR spectrum of VMT-PVC composite shows strong absorption at 2920 and $2849 \mathrm{~cm}^{-1}$, which is ascribed to the symmetrical stretching vibration of $-\mathrm{CH}_{3}$ and $-\mathrm{CH}_{2}$, respectively.

Fig. 2 shows TG/DTG/DSC curves of pure PVC and VMT-PVC composite with $6 \mathrm{wt} \%$ VMT nanosheets. The thermal decomposition of PVC apparently suffers two major mass loss stages. The first stage of mass loss from 200 to $400{ }^{\circ} \mathrm{C}$ can be assigned to the volatilisation of plasticizer and dehydrochlorination of PVC. In this stage, an estimated mass loss of $75.8 \%$ and $72.1 \%$ can be identified for PVC and VMT-PVC around $293.4{ }^{\circ} \mathrm{C}$ and $306.9{ }^{\circ} \mathrm{C}$, respectively. Along with the large mass loss, there are broad and weak endothermic peaks at $296.5^{\circ} \mathrm{C}$ and $308.7^{\circ} \mathrm{C}$ in the differential curves. The second mass loss stage occurred between $400{ }^{\circ} \mathrm{C}$ and $1000{ }^{\circ} \mathrm{C}$ is attributed to the slow pyrolysis reaction of PVC that ultimately leads to carbonaceous char residue, where a mass loss of $17.2 \%$ and $14.7 \%$ is observed for PVC and VMT-PVC, respectively. For the whole process, the total mass loss of the VMT-PVC decreased by $6.2 \%$ than pure PVC. Besides, the dehydrochlorination temperature appears to be increased by $13{ }^{\circ} \mathrm{C}$ for the VMT-PVC. These results suggest that the layered VMT plays an important role in resisting PVC dehydrochlorination.

The thermal stabilities of PVC and VMT-PVC with different VMT nanosheets are determined by the results of static thermal aging tests, as compared in Fig. 3a. It can be seen that the color of pure PVC becomes yellowish after $10 \mathrm{~min}$ at $180{ }^{\circ} \mathrm{C}$, and becomes black after $60 \mathrm{~min}$. The thermal stabilities of VMT-PVC samples are apparently improved. The onset
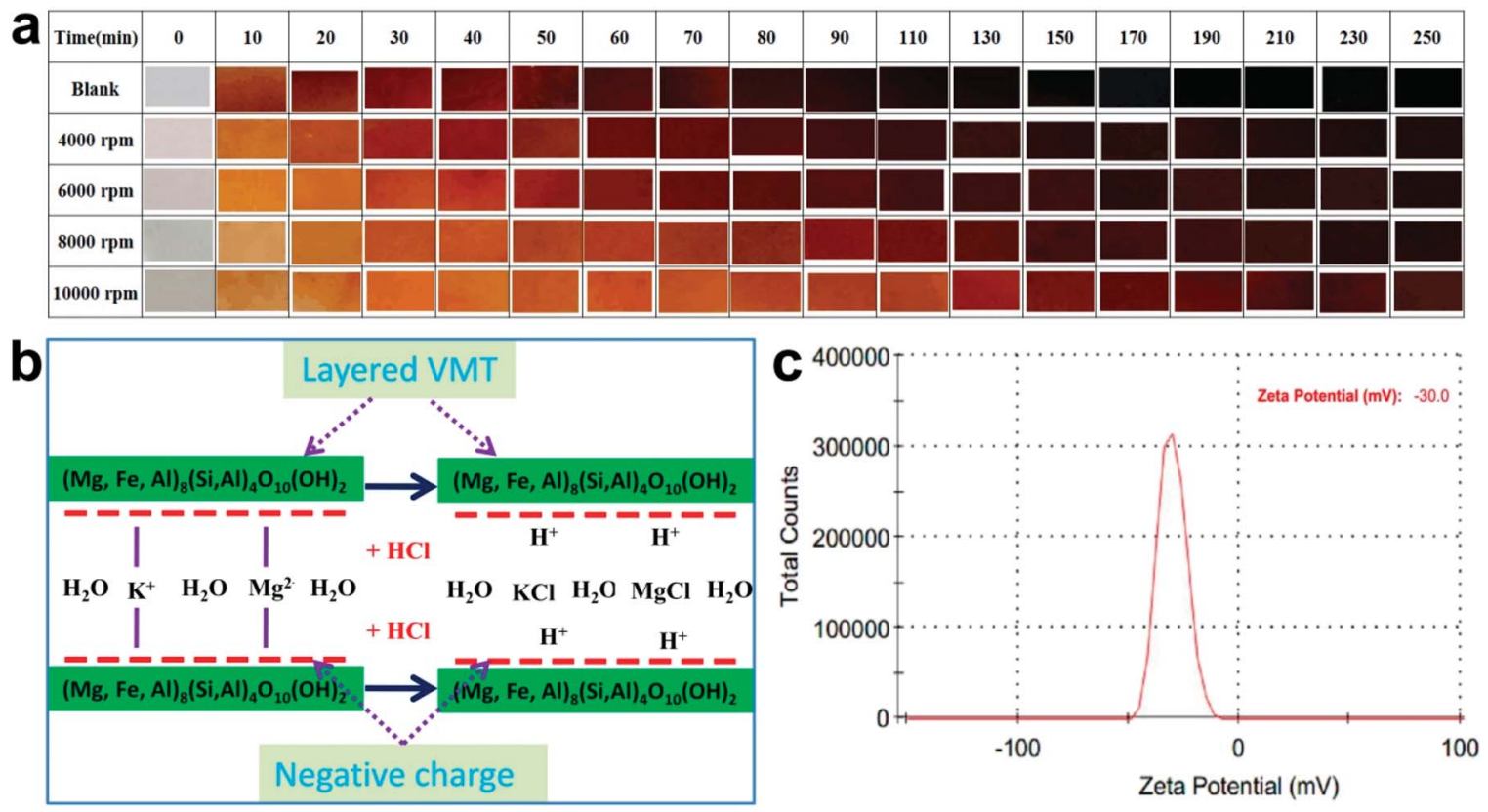

Fig. 3 (a) Color evolution of pure PVC and PVC samples stabilized by VMT nanosheets with different size (obtained under different separating rates). (b) Mechanism of layered VMT as thermal stabilizer of PVC. (c) Zeta potential for the dispersion of VMT nanosheets, showing charged feature of the VMT surface. 


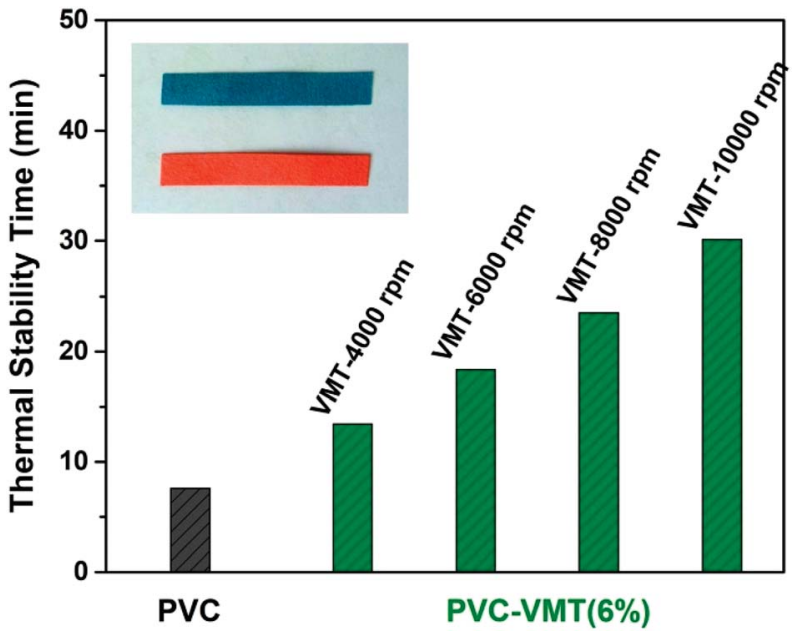

Fig. 4 Thermal stability time of pure PVC and PVC samples stabilized by VMT nanosheets with different size (obtained under different separating rates). Inset shows the color of Congo red test paper before and after test.

of blackening delays with reducing the particle size of VMT nanosheets. With VMT nanosheets obtained under separating rate of $10000 \mathrm{rpm}$, the VMT-PVC sample could maintain over $250 \mathrm{~min}$, which is better than La-doped hydrotalcite-like compounds. ${ }^{7}$ Besides, the VMT nanosheets exfoliated from mineral VMT possesses advantage of low-cost. Fig. $3 \mathrm{~b}$ indicates mechanism of layered VMT as thermal stabilizer in PVC. During thermal processing, the labile chlorine would be separated from the PVC chain. ${ }^{3}$ Substantially, the layered VMT possess negatively charged layers and interlayer metal cations. When the VMT is added in PVC, the negatively charged layers could absorb $\mathrm{H}^{+}$while the cations such as $\mathrm{K}^{+}$and $\mathrm{Mg}^{2+}$ could absorb the chlorine produced from PVC dehydrochlorination, which is given by the following formula:

$$
\mathrm{M}^{x-} \cdot \mathrm{A}^{n+}+\mathrm{HCl}=\mathrm{M}^{x-} \cdots x \mathrm{H}^{+}+\mathrm{A}^{n+} \cdots n \mathrm{Cl}^{-}
$$

where $\mathrm{M}^{x-} \cdot \mathrm{A}^{n+}$ is VMT, $\mathrm{M}^{x-}$ is VMT nanosheets, $\mathrm{A}^{n+}$ is interlayer metal cations, such as $\mathrm{K}^{+}, \mathrm{Ca}^{2+}$ and $\mathrm{Mg}^{2+}$. This could prevent $\mathrm{HCl}$ spillage, which is beneficial to the thermal stability of PVC. Fig. 3c shows surface zeta potential for aqueous dispersion of VMT nanosheets. The negative zeta potential of VMT nanosheets $(-30 \mathrm{mV})$, showing charged feature of the VMT surface. This result confirms the mechanism of layered VMT in stabilizing $\mathrm{HCl}$ produced from the thermal degradation of PVC. Fig. 4 shows the thermal stability time of PVC resin measured by the conventional Congo red test. The thermal stability time of the pure PVC is only about $8 \mathrm{~min}$, which increases after adding $6 \mathrm{wt} \%$ VMT nanosheets. By increasing the separating rate of VMT nanosheets from 4000 to $10000 \mathrm{rpm}$, the thermal stability time increases, which is in accordance with the results of static thermal aging tests (Fig. 3a). After addition of $6 \mathrm{wt} \%$ VMT nanosheets obtained under separating rate of $10000 \mathrm{rpm}$, the time extends to $30 \mathrm{~min}$, which is almost 4 times of the pure PVC.

\section{Conclusions}

In summary, well-defined VMT nanosheets were successfully exfoliated from mineral VMT by a facile water-assisted anionexchange approach. By delicately tailoring the adding amount of VMT nanosheets in the PVC resin, a perfect thermal stability of the PVC is accomplished. The dehydrochlorination temperature appears to be increased by $13{ }^{\circ} \mathrm{C}$ for the PVC resin with $6 \mathrm{wt} \%$ VMT additive. Furthermore, the effect of the thermal stability increased with reducing particle size, and nano-sized VMT shows equivalent thermal stability for PVC as industrially used hydrotalcite thermal stabilizers. The negatively charged laminates of VMT could absorb $\mathrm{H}^{+}$while the interlayer metal cations such as $\mathrm{K}^{+}$and $\mathrm{Mg}^{2+}$ could absorb $\mathrm{Cl}^{-}$produced from the dehydrochlorinated of PVC through covalent bond or hydrogen bond, which can help to prevent PVC degradation and $\mathrm{HCl}$ spillage. This work provides a promising approach to address the thermal stability problem in PVC resin, showing prospective in broad applications ranging from packaging to construction.

\section{Conflicts of interest}

There are no conflicts to declare.

\section{Acknowledgements}

This work was supported by National Natural Science Foundation of China (No. 21761029, 61604012), Open Laboratory of State Key Laboratory of Organic and Inorganic Composites (No. oic-201701008), Training Program for Outstanding Young Teachers in Xinjiang Corps, Joint Fund of Tarim University and Beijing University of Chemical Technology (No. ZZ-1501), First Division Alar Science and Technology Plan Project in Xinjiang Corps (2019GJJ04), Dean Project of Guangxi Key Laboratory of Petrochemical Resource Processing and Process Intensification Technology (No. 2017K005), and the Taishan Scholar Program of Shandong Province (No. tsqn201812055).

\section{Notes and references}

1 C. M. R. Abreu, A. C. Fonseca, N. M. P. Rocha, J. T. Guthrie, A. C. Serra and J. F. J. Coelho, Prog. Polym. Sci., 2018, 87, 3469.

2 J. Yu, L. Sun, C. Ma, Y. Qiao and H. Yao, Waste Manage., 2016, 48, 300-314.

3 Y. Shi, Y. Wang, B. Ma, M. Ma, S. Chen and X. Wang, J. Appl. Polym. Sci., 2019, 136, 47004.

4 W. H. Starnes Jr, Prog. Polym. Sci., 2002, 27, 2133-2170.

5 T. Dong, D. Li, Y. Li, W. Han, L. Zhang, G. Xie, J. Sunarso and S. Liu, Polym. Degrad. Stab., 2019, 159, 125-132.

6 K. M. Goodenough, F. Wall and D. Merriman, Nat. Resour. Res., 2017, 27, 201-216.

7 R. Wen, Z. Yang, H. Chen, Y. Hu and J. Duan, J. Rare Earths, 2012, 30, 895-902. 
8 I. Janica, S. D. Buffa, A. Mikolajczak, M. Eredia, D. Pakulski, A. Ciesielski and P. Samori, Nanoscale, 2018, 10, 2318223190.

9 M. Malandrino, O. Abollino, S. Buoso, A. Giacomino, C. La Gioia and E. Mentasti, Chemosphere, 2011, 82, 169-178.

10 M. A. Priolo, K. M. Holder, S. M. Greenlee and J. C. Grunlan, ACS Appl. Mater. Interfaces, 2012, 4, 5529-5533.

11 S. Takahashi, H. A. Goldberg, C. A. Feeney, D. P. Karim, M. Farrell, K. O'Leary and D. R. Paul, Polymer, 2006, 47, 3083-3093.

12 W. Tian, X. Kong, M. Jiang, X. Lei and X. Duan, Mater. Lett., 2016, 175, 110-113.
13 J. Y. Cheong, J. Ahn, M. Seo and Y. S. Nam, RSC Adv., 2015, 5, 61768-61774.

14 L. Yan, L. Fu, Y. Chen, H. Tian, A. Xiang and A. V. Rajulu, J. Appl. Polym. Sci., 2017, 134, 44828.

15 W. Tian, H. Li, B. Qin, Y. Xu, Y. Hao, Y. Li, G. Zhang, J. Liu, X. Sun and X. Duan, J. Mater. Chem. A, 2017, 5, 7103-7110.

16 K. Zhang, J. Xu, K. Y. Wang, L. Cheng, J. Wang and B. Liu, Polym. Degrad. Stab., 2009, 94, 2121-2127.

17 S. Bai, K. Zhang, R. Luo, D. Li, A. Chen and C. C. Liu, J. Mater. Chem., 2012, 22, 12643. 